\title{
Identification of Altered Growth Phenotypes in Human Breast Cancer Cells Using Cell Culture Methods That Support Growth of Normal and Neoplastic Mammary Epithelial Cells
}

\author{
Stephen P. Ethier, PhD, Cheryl M. Dilts, BS, Lori J. Pierce, MD, Kristine Kokeny, MD, \\ and Michael Mahacek, MD
}

The University of Michigan Medical Center, Department of Radiation Oncology, Ann Arbor, MI 48109-0582

\begin{abstract}
Over the past several years our laboratory has been studying factors that regulate proliferation of normal human mammary epithelial (HME) cells in order to better understand the alterations in cellular growth control mechanisms that occur during breast cancer development. To perform these experiments, we have either modified or developed cell culture methods for the isolation and growth of normal and neoplastic HME cells obtained from patient biopsy specimens. From these studies we have found that normal HME cells of the luminal lineage (the lineage from which breast cancer arises) have strict requirements for specific growth factor combinations for in vitro growth. Furthermore, these cells have a finite proliferative lifespan in culture. By contrast, human breast cancer (HBC) cells isolated from primary and metastatic sites exhibit many growth phenotypes that distinguish them from normal cells. First, whereas normal HME cells proliferate in culture with doubling times of 24-36 hours, HBC cells obtained from patient samples proliferate with doubling times of 100-200 hours. These proliferation kinetics are consistent with the rate at which these cells proliferate in vivo. This observation indicates that there are fundamental differences in growth regulation between normal and neoplastic mammary epithelial cells. Second, the majority of HBC cells isolated from human samples exhibit an extended proliferative lifespan in culture. Whereas normal HME cells undergo cell senescence after 15-20 population doublings, HBC cells often give rise to cell lines with indefinite proliferative potential. Third, HBC cells become independent of growth factors which are strictly required by normal HME cells for growth under defined conditions. In our experiments, escape from the requirements of exogenous epidermal growth factor (EGF) has been observed in cells from four patient-derived samples. Interestingly, the cellular mechanisms by which cells become EGF-independent for growth is different in cell lines isolated from different patients. Two breast cancer cell lines isolated in our laboratory proliferate continuously in serum-free, EGF-free medium and do not express EGF receptors. Thus, these cells are completely independent of EGF-mediated signalling pathways for their growth. A third cell line isolated in our laboratory has an amplified EGF receptor gene and overexpresses EGF receptor protein. Western blot analysis indicates that the tyrosine residues of the EGF receptor proteins in these cells are highly phosphorylated. These cells do not secrete any EGF-like growth factors that could be activating the receptors in an autocrine manner. This suggests that amplification and overexpression of EGF receptors can yield constitutively activated receptors that provide a mitogenic signal in the absence of a stimulatory ligand. Finally, we have analyzed the EGF requirements of human breast cancer cells that overexpress the erbB-2 receptor as a result of gene amplification. The results of these experiments indicated that overexpression of erbB-2 is, by itself, insufficient to overcome the EGF requirements of human breast cancer cells. However, one cell line that has a 15- to 20-fold amplification of erbB-2 and which expresses very high levels of tyrosine phosphorylated erbB-2 protein, is EGF-independent for growth. Thus, cell culture systems that allow proliferation of normal HME cells and HBC cells under well-defined culture conditions can result in identification of altered growth phenotypes associated with the neoplastic progression of breast cancer cells. In addition, isolation of cells exhibiting altered growth phenotypes may lead to insights as to the genetic mechanisms resulting in altered growth regulation in breast cancer cells. (c) 1993 Wiley-Liss, Inc.
\end{abstract}

